\title{
The Spend-It-All Region and Small Time Results for the Continuous Bomber Problem
}

\author{
Jay Bartroff ${ }^{1}$, Larry Goldstein ${ }^{1}$, and Ester Samuel-Cahn ${ }^{2}$ \\ ${ }^{1}$ Department of Mathematics, University of Southern California, Los Angeles, California, USA \\ ${ }^{2}$ Department of Statistics and Center for the Study of Rationality, \\ The Hebrew University of Jerusalem, Israel
}

\begin{abstract}
口
Abstract: A problem of optimally allocating partially effective ammunition $x$ to be used on randomly arriving enemies in order to maximize an aircraft's probability of surviving for time $t$, known as the Bomber Problem, was first posed by Klinger and Brown (1968). They conjectured a set of apparently obvious monotonicity properties of the optimal allocation function $K(x, t)$. Although some of these conjectures, and versions thereof, have been proved or disproved by other authors since then, the remaining central question, that $K(x, t)$ is nondecreasing in $x$, remains unsettled. After reviewing the problem and summarizing the state of these conjectures, in the setting where $x$ is continuous we prove the existence of a "spend-it-all" region in which $K(x, t)=x$ and find its boundary, inside of which the long-standing, unproven conjecture of monotonicity of $K(\cdot, t)$ holds. A new approach is then taken of directly estimating $K(x, t)$ for small $t$, providing a complete small- $t$ asymptotic description of $K(x, t)$ and the optimal probability of survival.
\end{abstract}

Keywords: Ammunition rationing; Optimal allocation; Poisson process; Sequential optimization.

Subject Classifications: 60G40; 62L05; 91A60.

\section{INTRODUCTION}

Klinger and Brown (1968) introduced a problem of optimally allocating partially effective ammunition to be used on enemies arriving at a Poisson rate in order to maximize the probability that an aircraft (hereafter "the bomber") survives for time $t$, known as the Bomber Problem. Given an amount $x$ of ammunition, let $K(x, t)$ denote the optimal amount of ammunition the bomber would use upon confronting an enemy at time $t$, defined as the time remaining to survive. The appearance of enemies is driven by a time-homogeneous Poisson process of known rate, taken to be 1 . An enemy survives the bomber's expenditure of an amount $y \in[0, x]$ of its ammunition with the geometric probability $q^{y}$, for some known $q \in(0,1)$, after which the enemy has a chance to destroy the bomber, which happens with known probability $v \in(0,1]$ (the $v=0$ case being trivial). By rescaling $x$, we assume without loss of generality that $q=e^{-1}$, and hence the probability that the bomber survives an enemy encounter in which it spends an amount $y$ of its ammunition is

$$
a(y)=1-v e^{-y} .
$$

Klinger and Brown (1968) posed two seemingly obvious conjectures about the optimal allocation function $K(x, t)$ :

A: $K(x, t)$ is nonincreasing in $t$ for all fixed $x \geq 0$;

Address correspondence to Jay Bartroff, Department of Mathematics, University of Southern California, KAP 108, Los Angeles, CA 90089, USA; E-mail: bartroff@usc.edu 
B: $K(x, t)$ is nondecreasing in $x$ for all fixed $t \geq 0$.

Klinger and Brown (1968) showed that [B] implies [A] when $v=1$, although, as will be discussed below, [B] remains in doubt. Improving the situation, Samuel (1970) showed that [A] holds without assuming [B] in the setting where units of ammunition $x$ are discrete, and in this setting also showed that a third conjecture holds:

C: The amount $x-K(x, t)$ held back by the bomber is nondecreasing in $x$ for all fixed $t \geq 0$.

[C] was first stated as a formal property by Simons and Yao (1990), who claimed that it can be shown to hold for continuous $x$ and $t$ by arguments similar to the ones they provide for a case where both $x$ and $t$ are discrete, and they also make theoretical and computational progress toward [B] in various discrete/continuous settings. Also in the setting where both $x$ and $t$ are continuous, Bartroff, Goldstein, Rinott, and Samuel-Cahn (2010) recently showed that [A] holds, and provide a full proof of [C] in this setting. Weber (1985) considered an infinite-horizon variant of the Bomber Problem in which the objective is to maximize the number of enemies shot down (thus removing $t$ from the problem) and found that, for discrete $x$, the property related to [B], that of monotonicity of $K(x)$, fails to hold. Shepp et al. (1991) considered the infinite-horizon problem for continuous $x$ and reached the same conclusion. On the other hand, Bartroff et al. (2010) consider the variation of the problem where the bomber is invincible, and both $x$ and $t$ are present and continuous, and show that $[\mathrm{B}]$ holds.

In spite of the results of Weber (1985), Shepp et al. (1991), and Bartroff et al. (2010), conjecture [B] has not been settled in any close relative to the original Bomber Problem, and it remains the conjecture about which the least is known. To gain insight into the function $K(x, t)$, perhaps as a step towards resolving [B] in greater generality, we take a new approach to the Bomber Problem of directly estimating, or when possible solving for, $K(x, t)$ when both $x$ and $t$ are continuous. One might expect $a$ priori that if $x$ or $t$ is sufficiently small then the optimal strategy is to spend all or nearly all of the available ammunition $x$, i.e., that $K(x, t)$ is equal to or nearly $x$. On the other hand, since the ammunition is assumed to be continuous it is not obvious that there exists a "spend-it-all" region where $K(x, t)$ is identically $x$. In Section 2 we show that there is indeed a spend-it-all region of $(x, t)$ values for which $K(x, t)=x$ and where [B] holds, and we estimate the region's boundary in Theorem 2.1, and are able to find it exactly in most cases. However, in Section 3 we show that there are many other regimes in which $K(x, t)$ is not so simple, but can nevertheless be described asymptotically for small values of $t$. In particular, in Theorem 3.1 we characterize the asymptotic behavior of $K(x, t)$ for small $t$ and show that regardless of how small $t$ is, there are large intervals of $x$ values for which $K(x, t) / x$ approaches any, even arbitrarily small, positive fraction, in stark contrast to the spend-itall strategy. The relation of these results to the outstanding conjecture [B] and extensions are discussed in Section 4 .

\section{THE SPEND-IT-ALL REGION}

In this section we describe an $(x, t)$-region where $K(x, t)$ is identically $x$, the so-called "spend-it-all" region. The boundary of this region is solved for, exactly as [2.1), except for a special configuration of the parameters $x, t, v$ in which the boundary is estimated from both sides; see (2.8). Bartroff (2010) has in the meantime shown that 2.1 always gives the exact boundary of the spend-it-all region.

In what follows, let $u=1-v \in[0,1)$ denote the probability that the bomber survives an enemy's counterattack, let $P(x, t)$ denote the optimal probability of survival at time $t$ when the bomber has ammunition $x$, and let $H(x, t)$ denote the optimal conditional probability of survival given an enemy at time $t$, with ammunition $x$. 
Theorem 2.1. For $u \in(0,1)$ and $t>0$ define

$$
f_{u}(t)=\log \left[1+u /\left(e^{t u}-1\right)\right],
$$

and extend this definition to $u=0$ by defining

$$
f_{0}(t)=\lim _{u \rightarrow 0} f_{u}(t)=\log \left(1+t^{-1}\right) .
$$

For $u \in[0,1)$ and $t>0$ define

$$
g_{u}(t)=\log \left(1+t^{-1}-u\right) .
$$

If $u \in[0,1)$ and $t>0$ satisfy one of the following:

$$
\begin{array}{ll}
\text { (i) } & u=0, \\
\text { (ii) } & u \in(0,1 / 2) \text { and } t \geq u^{-1} \log (2 v), \\
\text { (iii) } & u \in[1 / 2,1)
\end{array}
$$

then

$$
K(x, t)=x \text { if and only if } x \leq f_{u}(t) .
$$

In the remaining case, where

$$
u \in(0,1 / 2) \text { and } t<u^{-1} \log (2 v),
$$

we have

$$
K(x, t)=x \text { if } x \leq g_{u}(t) \text {, and } K(x, t)<x \text { if } x>f_{u}(t) .
$$

The theorem may be summarized by saying that, except for the configuration of $t, u$ values in (2.7), the spend-it-all region's boundary is given exactly by $f_{u}(t)$, which is positive for all $t>0$ and approaches 0 as $t \rightarrow \infty$. Bartroff (2010) has recently shown that $f_{u}(t)$ is the boundary of the spend-it-all region for all $t>0$ and $u \in[0,1)$. Here, in the remaining case $(2.7)$, the boundary is estimated from above by $f_{u}(t)$ and from below by $g_{u}(t)$, which is strictly less than $f_{u}(t)$ for all $t>0$ but asymptotically equivalent to it as $t \rightarrow 0$. Although $g_{u}(t)$ is negative for $t>u^{-1}$, it is utilized as a bound only when 2.7 holds, in which case $u^{-1}>u^{-1} \log (2 v)>0$. A consequence of the theorem is that, regardless of the value of $u$, for any $x>0$ there is $t$ sufficiently small such that the optimal strategy spends it all (i.e., $K(x, t)=x$ ), and for any $t>0$ there is $x$ sufficiently small such that the optimal strategy spends it all.

Proof. We first prove that $K(x, t)=x$ when $x$ is bounded from above by $f_{u}(t)$ and one of $(2.3)-(2.5)$ holds, or when $x$ is bounded from above by $g_{u}(t)$ and 2.7 holds. To begin, fix $x, t$ and let $u$ be any value in $[0,1)$. We make use of the crude upper bound on the optimal survival probability

$$
P(x, t) \leq \exp \left(-v t e^{-x}\right) \text { for all } \quad x, t>0,
$$

which corresponds to the infeasible strategy of firing an amount $x$ of ammunition at every possible enemy, giving

$$
P(x, t) \leq \sum_{i=0}^{\infty} e^{-t}[t a(x)]^{i} / i !=e^{-t} e^{t a(x)}=e^{-t(1-a(x))}=\exp \left(-v t e^{-x}\right) .
$$

Using (2.9), the optimal conditional survival probability is then

$$
H(x, t)=a(K(x, t)) P(x-K(x, t), t) \leq F(x-K(x, t)),
$$


where for fixed $x$ and $t$ we write

$$
F(y)=a(x-y) \exp \left(-v t e^{-y}\right) .
$$

By Lemma 2.1 below, $F$ is unimodal on $\mathbb{R}$ with maximum at

$$
y^{*}=\log \left(-v t+\sqrt{v^{2} t^{2}+4 t e^{x}}\right)-\log 2,
$$

which is not necessarily in $[0, x]$. In fact, if $x \leq g_{u}(t)$, then

$$
\begin{aligned}
y^{*} & \leq \log \left(-v t+\sqrt{v^{2} t^{2}+4 t e^{g_{u}(t)}}\right)-\log 2 \\
& =\log \left(-v t+\sqrt{v^{2} t^{2}+4 t\left(1+t^{-1}-u\right)}\right)-\log 2 \\
& =\log \left(-v t+\sqrt{(v t+2)^{2}}\right)-\log 2 \\
& =0,
\end{aligned}
$$

hence in this case $\max _{y \in[0, x]} F(y)=F(0)=a(x) e^{-t v}$. If it were that $K(x, t)<x$, then we would have

$$
H(x, t) \leq F(x-K(x, t))<F(0)=a(x) e^{-t v},
$$

a contradiction since the latter is the conditional survival probability of the spend-it-all strategy:

$$
a(x) \sum_{i=0}^{\infty} u^{i} e^{-t} t^{i} / i !=a(x) e^{-t} e^{t u}=a(x) e^{-t v} .
$$

Note that $e^{-t v}$ is the probability of not being killed in the enemy's thinned Poisson process with parameter $v$. The argument leading to 2.10 thus shows that $K(x, t)=x$ whenever $x \leq g_{u}(t)$; in particular, $K(x, t)=x$ when 2.7 holds, or when 2.3 holds after noting that $g_{0}(t)=f_{0}(t)$. For the remaining cases (2.4) and 2.5), we obtain a tighter bound. Fix $x, t$ and let $u \in(0,1)$. Letting

$$
G(y)=a(x-y) e^{-t}\left[1+e^{v y / u}\left(e^{t u}-1\right)\right]
$$

we claim that

$$
H(x, t) \leq G(x-K(x, t)) .
$$

To prove 2.12), first, a simple verification yields that for any nonnegative $b_{1}, \ldots, b_{i}$,

$$
\prod_{j=1}^{i} a\left(b_{j}\right) \leq a(y / i)^{n} \quad \text { when } \sum_{j=1}^{i} b_{j}=y .
$$

Hence, $H(x, t) \leq \widetilde{G}(x-K(x, t))$, where

$$
\widetilde{G}(y)=a(x-y) e^{-t}\left[1+\sum_{i=1}^{\infty} \frac{(t a(y / i))^{i}}{i !}\right],
$$

as the right hand side is the probability of survival for the infeasible strategy where one is given the number $i$ of future encounters, and divides the remaining amount $x-K(x, t)$ of ammunition optimally among them, firing $(x-K(x, t)) / i$ at each. Next, we claim that

$$
a(y / i)^{i} \leq u^{i} e^{v y / u} \quad \text { for all } y \in[0, x] \text { and all } i \geq 1,
$$


implying that $\widetilde{G}(y) \leq G(y)$ for all $y \in[0, x]$, and hence $\left[2.12\right.$. Letting $\rho_{i}=[a(y / i) / u]^{i}, 2.14$ is true since $\lim _{i \rightarrow \infty} \rho_{i}=e^{v y / u}$ and $\rho_{i}$ is evidently a nondecreasing sequence:

$$
\begin{aligned}
u^{i}\left(\rho_{i}-\rho_{i-1}\right) & =a(y / i)^{i}-u a(y /(i-1))^{i-1} \\
& =a(y / i)^{i}-a(0) a(y /(i-1))^{i-1} \\
& \geq 0,
\end{aligned}
$$

this last by (2.13). We will show below that if 2.4 ) or 2.5 holds, then $G(y)$ is uniquely maximized over $y \in[0, x]$ at $y=0$. Since $G(0)=a(x) e^{-t v}$, it then follows that $K(x, t)=x$, as above. To verify the maximum of $G$, we show that $G^{\prime}(0) \leq 0$ and $G^{\prime \prime}(y)<0$ for all $y \in(0, x]$. We compute

$$
\begin{aligned}
e^{t} G^{\prime}(y) & =-\frac{v}{u}\left\{e^{-x}\left[u e^{y}+e^{y / u}\left(e^{t u}-1\right)\right]-e^{v y / u}\left(e^{t u}-1\right)\right\} \\
e^{t} G^{\prime \prime}(y) & =-\frac{v}{u^{2}}\left\{e^{-x}\left[u^{2} e^{y}+e^{y / u}\left(e^{t u}-1\right)\right]-v e^{v y / u}\left(e^{t u}-1\right)\right\} .
\end{aligned}
$$

If $x \leq f_{u}(t)$, which is equivalent to $e^{-x} \geq\left(1+u /\left(e^{t u}-1\right)\right)^{-1}$, then we have

$$
\begin{aligned}
-\left(\frac{u}{v}\right) e^{t} G^{\prime}(0) & =e^{-x}\left(u+\left(e^{t u}-1\right)\right)-\left(e^{t u}-1\right) \\
& \geq\left(1+\frac{u}{e^{t u}-1}\right)^{-1}\left(e^{t u}-v\right)-\left(e^{t u}-1\right) \\
& =\left(\frac{e^{t u}-1}{e^{t u}-v}\right)\left(e^{t u}-v\right)-\left(e^{t u}-1\right) \\
& =0
\end{aligned}
$$

hence $G^{\prime}(0) \leq 0$. Next,

$$
\begin{aligned}
-\left(\frac{u^{2} e^{-v y / u}}{v}\right) e^{t} G^{\prime \prime}(y) & =e^{-x}\left[u^{2} e^{(2 u-1) y / u}+e^{y}\left(e^{t u}-1\right)\right]-v\left(e^{t u}-1\right) \\
& =e^{-x} p(y)-v\left(e^{t u}-1\right),
\end{aligned}
$$

where $p(y)=u^{2} e^{(2 u-1) y / u}+e^{y}\left(e^{t u}-1\right)$. When $u \geq 1 / 2$ the function $p(y)$ is clearly increasing in $y$ so for $y>0$ and $x \leq f_{u}(t)$

$$
\begin{aligned}
-\left(\frac{u^{2} e^{-v y / u}}{v}\right) e^{t} G^{\prime \prime}(y) & >e^{-x} p(0)-v\left(e^{t u}-1\right) \\
& =e^{-x}\left(u^{2}+e^{t u}-1\right)-v\left(e^{t u}-1\right) \\
& \geq\left(\frac{e^{t u}-1}{e^{t u}-v}\right)\left(u^{2}+e^{t u}-1\right)-v\left(e^{t u}-1\right) \\
& =\left(\frac{e^{t u}-1}{e^{t u}-v}\right)\left[u^{2}+e^{t u}-1-v\left(e^{t u}-v\right)\right] \\
& =\left(\frac{e^{t u}-1}{e^{t u}-v}\right)\left[u\left(e^{t u}-2 v\right)\right] \\
& \geq 0
\end{aligned}
$$

since $u \geq 1 / 2$ implies that $2 v \leq 1 \leq e^{t u}$. Finally, we show that when 2.4 holds, $p(y)$ is still increasing. First compute

$$
\begin{aligned}
& p^{\prime}(y)=u(2 u-1) e^{(2 u-1) y / u}+e^{y}\left(e^{t u}-1\right), \\
& p^{\prime \prime}(y)=(2 u-1)^{2} e^{(2 u-1) y / u}+e^{y}\left(e^{t u}-1\right) \quad>0
\end{aligned}
$$


and

$$
\begin{aligned}
p^{\prime}(0) & =u(2 u-1)+\left(e^{t u}-1\right) \\
& \geq u(2 u-1)+(2 v-1) \quad\left(\text { since } t \geq u^{-1} \log (2 v)\right) \\
& =2 u^{2}-3 u+1 \\
& =2(1-u)(1 / 2-u) \\
& >0
\end{aligned}
$$

since $u<1 / 2$. Thus, the steps leading to 2.15 hold in this case as well, completing the proof that $K(x, t)=x$ when 2.3, 2.4, 2.5, or 2.7) holds.

To complete the proof of the theorem, we show that $K(x, t)<x$ when $x>f_{u}(t)$. To do this, we bound $H(x, t)$ from below by the conditional survival probability $\underline{H}(y)$ of the strategy that fires an amount $y \in[0, x]$ of ammunition at the present enemy, fires all remaining ammunition $x-y$ at the next enemy (if one is encountered), and hopes for the best thereafter. First assume that $u \in(0,1)$ and fix $x, t$ satisfying $x>f_{u}(t)$. Then

$$
\begin{aligned}
\underline{H}(y) & =a(y)\left[e^{-t}+e^{-t} \sum_{i=1}^{\infty} \frac{t^{i} a(x-y) u^{i-1}}{i !}\right] \\
& =a(y)\left[e^{-t}+e^{-t} \frac{a(x-y)}{u}\left(e^{t u}-1\right)\right] \\
& =e^{-t} a(y)\left[1+\left(\frac{e^{t u}-1}{u}\right) a(x-y)\right] .
\end{aligned}
$$

By applying Lemma 2.1 with $A=\left(e^{t u}-1\right) / u$, we see that $\underline{H}(y)$ is unimodal with maximum at $K^{*}(x, t)=$ $\left(x+f_{u}(t)\right) / 2$, which, since $x>f_{u}(t)$, satisfies $K^{*}(x, t)<(x+x) / 2=x$. If it were that $K(x, t)=x$, then we would have

$$
H(x, t)=a(x) e^{-t v}=\underline{H}(x)<\underline{H}\left(K^{*}(x, t)\right),
$$

a contradiction. If $u=0$, the conditional survival probability of this strategy is

$$
\underline{H}(y)=a(y)\left[e^{-t}+e^{-t} t a(x-y)\right]=e^{-t} a(y)[1+t a(x-y)],
$$

and a similar argument applies: By Lemma 2.1 with $A=t$, the function $\underline{H}(y)$ is unimodal with maximum at $K^{*}(x, t)=\left(x+f_{0}(t)\right) / 2<x$, leading to the same contradiction.

Lemma 2.1. Fix $x>0, t>0$, and $v \in(0,1]$. The function

$$
y \mapsto a(x-y) \exp \left(-v t e^{-y}\right)
$$

is unimodal on $\mathbb{R}$ with maximum at

$$
y^{*}=\log \left(-v t+\sqrt{v^{2} t^{2}+4 t e^{x}}\right)-\log 2 .
$$

For any fixed $A>0$, the function

$$
y \mapsto a(y)[1+A a(x-y)]
$$

is unimodal on $\mathbb{R}$ with maximum at

$$
y^{*}=\left[x+\log \left(1+A^{-1}\right)\right] / 2 .
$$


Proof. Taking the derivative of 2.16 with respect to $y$ and setting $z=e^{y}$ gives

$$
-v e^{-x-y} \exp \left(-v t e^{-y}\right)\left(e^{2 y}+v t e^{y}-t e^{x}\right)=-v e^{-x-y} \exp \left(-v t e^{-y}\right)\left(z^{2}+v t z-t e^{x}\right) .
$$

Since $z>0$, the function 2.16 increases in $y=\log z$ up to the $\log$ of the positive root of the quadratic in 2.20), which is (2.17), and decreases thereafter. Similarly, the derivative of 2.18) with respect to $y$ is

$$
-v\left(A e^{-x+y}-(1+A) e^{-y}\right)=-v e^{-y}\left(A e^{-x} z^{2}-(1+A)\right),
$$

and solving for the root gives 2.19.

\section{AN ASYMPTOTIC CHARACTERIZATION OF $K(x, t)$}

In this section we give an asymptotic description of the optimal allocation function $K(x, t)$ as $t \rightarrow 0$, and for this it suffices to consider sequences $(x, t)$ with $t \rightarrow 0$. In addition to giving an asymptotic description of the optimal survival probability $P(x, t)$ and the optimal conditional survival probability $H(x, t)$, our main goal is to characterize the fraction $K(x, t) / x$ of the current ammunition $x$ spent by the optimal strategy at time $t$, and it turns out that $K(x, t) / x$ approaches a finite nonzero limit on sequences $(x, t)$ such that $|\log t| / x$ approaches a finite nonzero limit. We thus give an essentially complete asymptotic description of $K(x, t)$ by considering sequences $(x, t)=\left(x_{t}, t\right)$ such that

$$
\frac{|\log t|}{x} \rightarrow \rho \in(0, \infty) \quad \text { as } t \rightarrow 0
$$

leaving divergent sequences to be handled by considering subsequences. We will write $x=x_{t}$ when we wish to emphasize the dependence of $x$ on $t$, but most of the time this notation will be suppressed. Note that a consequence of 3.1 is that $x \rightarrow \infty$ at the same rate at which $|\log t| \rightarrow \infty$ as $t \rightarrow 0$. It should perhaps not be surprising that this is the nontrivial asymptotic regime since the boundary of the spend-it-all region found in Theorem 2.1 is asymptotically equivalent to $|\log t|$ as $t \rightarrow 0$. In what follows, let $\left(\begin{array}{l}1 \\ 2\end{array}\right)^{-1}=\infty$.

Theorem 3.1. Under (3.1), let $j \in\{1,2, \ldots\}$ be such that

$$
\left(\begin{array}{c}
j+1 \\
2
\end{array}\right)^{-1} \leq \rho<\left(\begin{array}{l}
j \\
2
\end{array}\right)^{-1} .
$$

Then, as $t \rightarrow 0$,

$$
\begin{aligned}
\frac{K(x, t)}{x} & \rightarrow 1 / j+\rho(j-1) / 2 \\
\frac{1}{x}|\log (1-H(x, t))| & \rightarrow 1 / j+\rho(j-1) / 2 \\
\frac{1}{x}|\log (1-P(x, t))| & \rightarrow 1 / j+\rho(j+1) / 2 .
\end{aligned}
$$

The theorem is proved in the next subsection. First, we briefly discuss the result. Note that the $j$ satisfying (3.2) is nonincreasing in $\rho$ and, in particular, $\rho \geq 1$ corresponds to $j=1$ while $\rho<1$ corresponds to $j>1$. The right hand sides of (3.3) and (3.4) equal 1 for $j=1$, and are in the interval $[2 /(j+1), 2 / j)$ for $j \geq 2$; similarly, the right hand side of 3.5 is in the interval $[2 / j, 2 /(j-1))$ for all $j \geq 1$. In particular, 3.3. implies that $K(x, t) / x$ can take on any value in $(0,1]$. The rates of convergence in $(3.3)-(3.5)$ are functions of the rate of convergence in 3.1. Specifically, without assuming more than $|\log t|-\rho x=o(x)$ in 3.1 , 




Figure 1. The small- $t$ asymptotic approximation (3.6) of $K(x, t)$.

the same $o(x)$ term appears in the convergence of $K(x, t),|\log (1-H(x, t))|$, and $|\log (1-P(x, t))|$ in (3.3)-(3.5). However, when $\rho>1$, the convergence is $O(1 / x)$ in (3.3) and (3.4), but in no other cases, an artifact of the natural upper bound $K(x, t) \leq x$ that is relevant only in the $\rho>1$ case.

The result (3.3) can equivalently be stated as, under (3.1),

$$
K(x, t) \sim \frac{x}{j}+\left(\frac{j-1}{2}\right)|\log t|
$$

as $t \rightarrow 0$ for $j$ satisfying (3.2). Hence, for small $t$, the first quadrant of the $(x, t)$-plane can be thought of as partitioned into the regions

$$
R_{j}=\left\{(x, t): \quad x>0, \quad t>0, \quad\left(\begin{array}{c}
j+1 \\
2
\end{array}\right)^{-1} \leq \frac{|\log t|}{x}<\left(\begin{array}{l}
j \\
2
\end{array}\right)^{-1}\right\}, \quad j=1,2, \ldots,
$$

which determine the asymptotic behavior of the optimal strategy. Figure 1 plots 3.6 and the boundaries of the first few $R_{j}$. Note that although (3.6) varies smoothly within each $R_{j}$, it is continuous but not smooth at the lower boundary of $R_{j}$. For small $t, K(x, t)$ given by 3.3 turns out to be such that if $(x, t) \in R_{j}$, then after firing $K(x, t)$ at an immediate enemy, the new state $(x-K(x, t), t)$ lies in $R_{j-1}$. This leads to the inductive method of proof, given in the next section. The boundary of the $R_{1}$ region is asymptotically equivalent to the estimates of the spend-it-all region's boundary in Theorem 2.1 in the strong sense that their difference is $o(1)$ as $t \rightarrow 0$. 


\subsection{Proof of Theorem 3.1}

We proceed by induction on $j$. To begin, assume that $j=1$. The optimal conditional survival probability $H(x, t)$ is bounded below by the conditional survival probability of the spend-it-all strategy (2.11), giving

$$
\begin{aligned}
H(x, t) & \geq a(x) e^{-t v} \\
& \geq\left(1-v e^{-x}\right)(1-t v) \\
& =1-v e^{-x}-v e^{-\rho x+o(x)}+v^{2} e^{-(\rho+1) x+o(x)} .
\end{aligned}
$$

Moving to $P(x, t)$, it is bounded below by the survival probability of the strategy that fires $x$ at the first enemy. Under such a strategy, the bomber survives if no enemy plane arrives during time $t$, which happens with probability $e^{-t}$, or if the bomber encounters and survives one enemy, which happens with probability $t e^{-t} a(x)$, and ignoring other enemy encounters we obtain

$$
\begin{aligned}
P(x, t) & \geq e^{-t}[1+t a(x)] \\
& \geq(1-t)\left[1+t-v t e^{-x}\right] \\
& =1-v t e^{-x}-t^{2}+v t^{2} e^{-x} \\
& =1-v e^{-(\rho+1) x+o(x)}-e^{-2 \rho x+o(x)}+v e^{-(2 \rho+1) x+o(x)} \\
& \geq 1-v e^{-(\rho+1) x+o(x)} .
\end{aligned}
$$

On the other hand, $P(x, t)$ is bounded above by the survival probability of the infeasible strategy that fires $x$ at the first enemy and, upon survival of this encounter, is guaranteed survival thereafter, so

$$
\begin{aligned}
P(x, t) & \leq e^{-t}\left[1+a(x)\left(e^{t}-1\right)\right] \\
& =1-v e^{-x}+v e^{-x} e^{-t} \\
& \leq 1-v e^{-x}+v e^{-x}\left(1-t+t^{2} / 2\right) \\
& =1-v t e^{-x}+v t^{2} e^{-x} / 2 \\
& =1-v e^{-(\rho+1) x+o(x)}+v e^{-(2 \rho+1) x+o(x)} / 2 \\
& =1-e^{-(\rho+1) x+o(x)} .
\end{aligned}
$$

For this $j=1$ case, we consider separately the cases $\rho=1$ and $\rho \in(1, \infty)$. First assume that $\rho>1$. In this case, $(3.8)$ is $1-v e^{-x}(1+o(1))$, and as the conditional probability of the spend-it-all strategy is bounded above by $a(K(x, t)) \cdot 1$, the probability of surviving the first encounter when expending the optimal amount $K(x, t)$ and ignoring future danger, we find that $1-v e^{-x}(1+o(1)) \leq 1-v e^{-K(x, t)}$ so that $x+o(1) \leq K(x, t) \leq x$. To estimate $H(x, t)$, plug $K(x, t)=x+o(1)$ into $a(K(x, t))$ to get

$$
a(K(x, t))=1-v e^{-x}(1+o(1)) .
$$

This is the same order as the lower bound 3.8, hence $H(x, t)=1-v e^{-x}(1+o(1))$, which implies 3.4 ) in this case. The limit (3.5) holds as well in this case since both (3.9) and $(3.10)$ are of order

$$
1-e^{-(\rho+1) x+o(x)}
$$

Since $H(x, t)=1-v e^{-x}(1+o(1))$ is equivalent to

$$
\frac{1}{x}|\log (1-H(x, t))|=1-(\log v) / x+o(1 / x)=1+O(1 / x),
$$

the error term on the right hand side of (3.4) in this case is $O(1 / x)$; this holds for 3.3 and 3.4 when $\rho>1$, but for no other cases. 
Now let $\rho=1$. The lower bound $(3.8)$ is

$$
1-2 v e^{-x+o(x)}=1-e^{-x+o(x)},
$$

and by Lemma 3.2 below we have $x+o(x) \leq K(x, t) \leq x$. Plugging $K(x, t)=x+o(x)$ into the upper bound $a(K(x, t))$ gives $H(x, t) \leq 1-e^{-x+o(x)}$, the same order as the lower bound 3.11 , , hence

$$
\frac{1}{x}|\log (1-H(x, t))| \rightarrow 1 .
$$

The lower bound $(3.9)$ gives

$$
P(x, t) \geq 1-e^{-2 x+o(x)},
$$

and the upper bound 3.10 gives the same order, hence

$$
\frac{1}{x}|\log (1-P(x, t))| \rightarrow 2=\rho+1 .
$$

This concludes the $j=1$ case.

Now let $I_{j}$ denote the half-closed interval (3.2), i.e.,

$$
I_{j}=\left[\left(\begin{array}{c}
j+1 \\
2
\end{array}\right)^{-1},\left(\begin{array}{l}
j \\
2
\end{array}\right)^{-1}\right),
$$

and let $\alpha_{j}(\rho)$ and $\beta_{j}(\rho)$ denote the right hand sides of 3.3 and 3.5 , respectively, i.e.,

$$
\begin{aligned}
\alpha_{j}(\rho) & =1 / j+\rho(j-1) / 2 \\
\beta_{j}(\rho) & =1 / j+\rho(j+1) / 2 .
\end{aligned}
$$

For the inductive step, assume that (3.3)- $(3.5)$ hold for $j$ and let $\rho$ belong to $I_{j+1} . H(x, t)$ is bounded below by the conditional survival probability of the strategy $\underline{H}(x, t)$ that fires $\widetilde{K}(x)=\alpha_{j+1}(\rho) x$ at the first enemy, and then behaves optimally thereafter. Letting

$$
x^{\prime}:=x-\widetilde{K}(x)=x\left[1-\alpha_{j+1}(\rho)\right],
$$

we have

$$
\rho^{\prime}:=\lim _{t \rightarrow 0} \frac{|\log t|}{x^{\prime}}=\frac{\rho}{1-\alpha_{j+1}(\rho)} \in I_{j}
$$

by Lemma 3.1 below. Then, by the inductive hypothesis, we have

$$
\underline{H}(x, t)=a(\widetilde{K}(x)) P\left(x^{\prime}, t\right)=\left[1-v e^{-\alpha_{j+1}(\rho) x}\right]\left[1-e^{-\beta_{j}\left(\rho^{\prime}\right) x^{\prime}+o\left(x^{\prime}\right)}\right],
$$

and

$$
\beta_{j}\left(\rho^{\prime}\right) \frac{x^{\prime}}{x}=\beta_{j}\left(\rho^{\prime}\right)\left[1-\alpha_{j+1}(\rho)\right]=\alpha_{j+1}(\rho)
$$

by Lemma 3.1 , giving

$$
\underline{H}(x, t)=\left[1-e^{-\alpha_{j+1}(\rho) x+o(x)}\right]^{2}=1-e^{-\alpha_{j+1}(\rho) x+o(x)} .
$$

Lemma 3.2 then implies that

$$
K(x, t) \geq \alpha_{j+1}(\rho) x+o(x)
$$


and we will show that this expression actually holds with equality. To do this, we consider sequences $(x, t)$ still for which $|\log t| / x \rightarrow \rho \in I_{j+1}$ and on which

$$
\tau:=\lim _{t \rightarrow 0} \frac{K(x, t)}{x}
$$

exists, and we will show that $\tau=\alpha_{j+1}(\rho)$ is the only possible limit. This suffices to show that the lim sup and lim inf of $K(x, t) / x$ both equal $\alpha_{j+1}(\rho)$.

By 3.17), we know that the only possible values of $\tau$ lie in $\left[\alpha_{j+1}(\rho), 1\right]$. First, suppose that there is a sequence $(x, t)$ on which $\tau \in\left(\alpha_{j+1}(\rho), 1\right)$. Then

$$
x^{\prime \prime}:=x-K(x, t) \sim(1-\tau) x \quad \text { and } \quad \rho^{\prime \prime}:=\lim _{t \rightarrow 0} \frac{|\log t|}{x^{\prime \prime}}=\frac{\rho}{1-\tau}>\frac{\rho}{1-\alpha_{j+1}(\rho)}=\rho^{\prime} \in I_{j}
$$

by Lemma 3.1, so let $i \in\{1,2, \ldots, j\}$ be such that $\rho^{\prime \prime} \in I_{i}$. Then, again by the inductive hypothesis, we would have

$$
H(x, t)=a(K(x, t)) P\left(x^{\prime \prime}, t\right)=\left[1-v e^{-\tau x+o(x)}\right]\left[1-e^{-\beta_{i}\left(\rho^{\prime \prime}\right) x^{\prime \prime}+o\left(x^{\prime \prime}\right)}\right],
$$

and

$$
\begin{aligned}
\beta_{i}\left(\rho^{\prime \prime}\right) \frac{x^{\prime \prime}}{x}=\left[\frac{1}{i}+\frac{\rho^{\prime \prime}(i+1)}{2}\right] \frac{x^{\prime \prime}}{x} & \rightarrow\left[\frac{1}{i}+\frac{\rho(i+1)}{2(1-\tau)}\right](1-\tau) \\
& =\frac{1-\tau}{i}+\frac{\rho(i+1)}{2} .
\end{aligned}
$$

If $i<j$, then $\rho /(1-\tau) \in I_{i}$ implies that $(1-\tau) \leq \rho\left(\begin{array}{c}i+1 \\ 2\end{array}\right)$, so 3.19 becomes

$$
\begin{aligned}
\frac{1-\tau}{i}+\frac{\rho(i+1)}{2} & \leq \rho(i+1) \\
& =\rho(i+1-j / 2)+\rho j / 2 \\
& \left.<\left(\begin{array}{c}
j+1 \\
2
\end{array}\right)^{-1}(j-j / 2)+\rho j / 2 \quad \text { (since } \rho \in I_{j+1} \text { and } i<j\right) \\
& =\alpha_{j+1}(\rho) .
\end{aligned}
$$

If $i=j$, then 3.19 becomes

$$
\begin{aligned}
\frac{1-\tau}{j}+\frac{\rho(j+1)}{2} & <\frac{1-\alpha_{j+1}(\rho)}{j}+\frac{\rho(j+1)}{2} \\
& =\alpha_{j+1}(\rho)-\frac{\left[(j+1) \alpha_{j+1}(\rho)-1\right]}{j}+\frac{\rho(j+1)}{2} \\
& =\alpha_{j+1}(\rho) .
\end{aligned}
$$

In both cases we have shown that 3.19 is less than $\alpha_{j+1}(\rho)<\tau$, which implies that 3.18 is

$$
1-\exp [-((1-\tau) / i+\rho(i+1) / 2) x+o(x)]
$$

and is hence smaller than 3.16 for small $t$, a contradiction.

Now assume that there is a sequence $(x, t)$ on which $\tau=1$. Using the crude bound $a(K(x, t)) \leq 1$ and 2.9 ,

$$
\begin{aligned}
H(x, t) & =a(K(x, t)) P(x-K(x, t), t) \\
& \leq 1 \cdot \exp \left[-t v e^{-(x-K(x, t))}\right] \\
& \leq 1-v t e^{-(x-K(x, t))}+v^{2} t^{2} e^{-2(x-K(x, t))} / 2 \\
& =1-v e^{-\rho x+o(x)}+v^{2} e^{-2 \rho x+o(x)} \\
& =1-e^{-\rho x+o(x)}
\end{aligned}
$$


which leads to the same contradiction since $\rho<\alpha_{j+1}(\rho)$ by Lemma 3.1. We have shown that $\alpha_{j+1}(\rho)$ is the only possible value of $\tau$, hence (3.17) holds with equality and 3.16) holds for $H(x, t)$. All that remains is to verify (3.5) for the $j+1$ case.

Let $T$ denote the exponentially distributed waiting time, with mean 1, until the first enemy, and recall that we write $x=x_{t}$ to emphasize the dependence on $t$. Then $P$ and $H$ are related through the expectation

$$
\begin{aligned}
P(x, t)=P\left(x_{t}, t\right) & =E\left[H\left(x_{t}, t-T\right) \mathbf{1}\{T<t\}+\mathbf{1}\{T \geq t\}\right] \\
& =\int_{0}^{t} H\left(x_{t}, t-r\right) e^{-r} d r+P(T \geq t) \\
& =e^{-t}\left[\int_{0}^{t} H\left(x_{t}, s\right) e^{s} d s+1\right]
\end{aligned}
$$

Using 3.20 and that $H(x, \cdot)$ is nonincreasing, we have

$$
\begin{aligned}
P(x, t) & \geq e^{-t}\left[H\left(x_{t}, t\right) \int_{0}^{t} e^{s} d s+1\right] \\
& =e^{-t}\left[H\left(x_{t}, t\right)\left(e^{t}-1\right)+1\right] \\
& =e^{-t}\left[1-H\left(x_{t}, t\right)\right]+H\left(x_{t}, t\right) \\
& \geq(1-t)\left[1-H\left(x_{t}, t\right)\right]+H\left(x_{t}, t\right) \\
& =1-t\left[1-H\left(x_{t}, t\right)\right] \\
& =1-e^{-\rho x+o(x)} e^{-\alpha_{j+1}(\rho) x+o(x)} \\
& =1-e^{-\left[\rho+\alpha_{j+1}(\rho)\right] x+o(x)} \\
& =1-e^{-\beta_{j+1}(\rho) x+o(x)}
\end{aligned}
$$

by Lemma 3.1. We bound $P(x, t)$ from above by a function of the same order. Fix $\delta \in(0,1)$ and note that

$$
\frac{|\log (\delta t)|}{x_{t}}=\frac{-\log (\delta t)}{x_{t}}=\frac{-\log t}{x_{t}}+\frac{-\log \delta}{x_{t}}=\frac{|\log t|}{x_{t}}+\frac{|\log \delta|}{x_{t}} \rightarrow \rho .
$$

Then, by (3.20),

$$
\begin{aligned}
P(x, t) & \leq e^{-t}\left[\int_{0}^{\delta t} e^{s} d s+H\left(x_{t}, \delta t\right) \int_{\delta t}^{t} e^{s} d s+1\right] \\
& =e^{-(1-\delta) t}\left[1-H\left(x_{t}, \delta t\right)\right]+H\left(x_{t}, \delta t\right) \\
& \left.\leq 1-(1-\delta) t+t^{2}\right]\left[1-H\left(x_{t}, \delta t\right)\right]+H\left(x_{t}, \delta t\right) \\
& =1-(1-\delta) t\left[1-H\left(x_{t}, \delta t\right)\right]+t^{2}\left[1-H\left(x_{t}, \delta t\right)\right] \\
& =1-(1-\delta) e^{-\rho x+o(x)} e^{-\alpha_{j+1}(\rho) x+o(x)}+e^{-2 \rho x+o(x)} e^{-\alpha_{j+1}(\rho) x+o(x)} \quad \text { (by (3.21)) } \\
& =1-e^{-\left[\rho+\alpha_{j+1}(\rho)\right] x+o(x)} \\
& =1-e^{-\beta_{j+1}(\rho) x+o(x)},
\end{aligned}
$$

completing the proof of Theorem 3.1 except for the following lemmas. The first collects various facts relating $\alpha_{j}(\rho), \beta_{j}(\rho)$, and $\rho$, and the second provides a crude but useful bound on $K(x, t)$. 
Lemma 3.1. Let $I_{j}, \alpha_{j}(\rho)$, and $\beta_{j}(\rho)$ be as in 3.12)-(3.14). Assume that $\rho \in I_{j+1}$ for some $j \geq 1$, and let $\rho^{\prime}=\rho /\left[1-\alpha_{j+1}(\rho)\right]$. Then

$$
\begin{aligned}
\rho & <\alpha_{j+1}(\rho), \\
\rho^{\prime} & \in I_{j}, \\
\beta_{j}\left(\rho^{\prime}\right) & =\left[1 / \alpha_{j+1}(\rho)-1\right]^{-1} . \\
\alpha_{j+1}(\rho)+\rho & =\beta_{j+1}(\rho)
\end{aligned}
$$

Proof. Let $\rho \in I_{j+1}$. Then

$$
\begin{aligned}
\beta_{j}\left(\rho^{\prime}\right) & =\frac{1}{j}+\frac{\rho^{\prime}(j+1)}{2} \\
& =\frac{1}{j}+\frac{2(j+1)^{2}}{2 j(2 / \rho-(j+1))} \\
& =\frac{\alpha_{j+1}(\rho)}{1-\alpha_{j+1}(\rho)}
\end{aligned}
$$

after some simplifying, proving 3.24). For 3.22,

$$
\begin{aligned}
\rho & =\rho(1-j / 2)+\rho j / 2 \\
& \leq \begin{cases}\rho / 2+\rho / 2, & j=1 \\
\rho j / 2, & j \geq 2\end{cases} \\
& < \begin{cases}1 / 2+\rho / 2, & j=1 \\
1 /(j+1)+\rho j / 2, & j \geq 2\end{cases} \\
& =\alpha_{j+1}(\rho) .
\end{aligned}
$$

For 3.23,

$$
\begin{aligned}
\rho^{\prime} & =\frac{2(j+1)}{j[2 / \rho-(j+1)]} \\
& \in\left[\frac{2(j+1)}{j\left[2\left(\begin{array}{c}
j+2 \\
2
\end{array}\right)-(j+1)\right]}, \frac{2(j+1)}{j\left[2\left(\begin{array}{c}
j+1 \\
2
\end{array}\right)-(j+1)\right]}\right) \\
& =\left[\left(\begin{array}{c}
j+1 \\
2
\end{array}\right)^{-1},\left(\begin{array}{l}
j \\
2
\end{array}\right)^{-1}\right)=I_{j} .
\end{aligned}
$$

For 3.25, $\alpha_{j+1}(\rho)+\rho=1 /(j+1)+\rho(j+2) / 2=\beta_{j+1}(\rho)$.

Lemma 3.2. If there is a $\gamma \in(0,1]$ such that $H(x, t) \geq 1-e^{-\gamma x+o(x)}$, then $K(x, t) \geq \gamma x+o(x)$.

Proof. We have

$$
H(x, t)=a(K(x, t)) P(x-K(x, t), t) \leq a(K(x, t)) \cdot 1=1-v e^{-K(x, t)},
$$

and setting this last $\geq$ the assumed lower bound $1-e^{-\gamma x+o(x)}$ leads to $K(x, t) \geq \gamma x+o(x)$. 


\section{DISCUSSION}

In Section 3 an inductive method is used to estimate the limiting optimal fraction $K(x, t) / x$ of ammunition used as $t \rightarrow 0$. The same result holds when the bomber is restricted to only firing discrete units (integers, say) of ammunition $x$, the only modification of the proof needed is to replace $x$ by $\lfloor x\rfloor$ (the largest integer $\leq x)$ in the appropriate places. For example, in the $\rho>1$ case in the proof of Theorem 3.1, we have $H(x, t) \geq a(\lfloor x\rfloor) e^{t v}$, which leads to $\lfloor x\rfloor+o(1) \leq K(x, t) \leq\lfloor x\rfloor$, and hence $K(x, t) / x \rightarrow 1$, using that $\lfloor x\rfloor / x \rightarrow 1$.

Theorem 2.1 shows that $K(x, t)=x$ in a region asymptotically equivalent to $R_{1}$ in 3.7 and, this being monotone in $x$, that conjecture [B] holds in this region. It is therefore natural to ask if the estimates of $K(x, t)$ in $R_{j}$ given by Theorem 3.1 can be used to shed any light on conjecture [B] for $j \geq 2$. One thing we can say is that [B] is satisfied in the limit as $t \rightarrow 0$ in the following sense. Letting $x_{1} \leq x_{2}$ be such that $\lim _{t \rightarrow 0}|\log t| / x_{1} \in R_{j}$ and $\lim _{t \rightarrow 0}|\log t| / x_{2} \in R_{j^{\prime}}$ for some $j \leq j^{\prime}$, by 3.6) we have

$$
K\left(x_{2}, t\right)-K\left(x_{1}, t\right) \sim \frac{x_{2}}{j^{\prime}}+\left(\frac{j^{\prime}-1}{2}\right)|\log t|-\left[\frac{x_{1}}{j}+\left(\frac{j-1}{2}\right)|\log t|\right] .
$$

If $j=j^{\prime}$, then 4.1) is $\left(x_{2}-x_{1}\right) / j \geq 0$. If $j<j^{\prime}$, then 4.1) divided by $|\log t|$ is

$$
\begin{aligned}
\frac{x_{2}}{j^{\prime}|\log t|}-\frac{x_{1}}{j|\log t|}+\frac{j^{\prime}-j}{2} & >\left(\frac{\left(\begin{array}{c}
j^{\prime} \\
2
\end{array}\right)}{j^{\prime}}-\frac{\left(\begin{array}{c}
j+1 \\
2
\end{array}\right)}{j}+\frac{j^{\prime}-j}{2}\right)(1+o(1)) \\
& =\left(j^{\prime}-j-1\right)(1+o(1))
\end{aligned}
$$

which approaches a nonnegative limit. However, to make this argument hold for, say, all $x_{1} \leq x_{2}$ sufficiently large and all $t$ sufficiently small, higher order asymptotics are needed. In particular, the rate of convergence in (3.3) as a function of $x$ and $t$ is needed, for which the tools developed in Sections 2 and 3 may be a starting point.

\section{ACKNOWLEDGEMENTS}

We thank Yosef Rinott for many helpful discussions on this topic. Bartroff's work was supported by grant DMS-0907241 from the National Science Foundation. 


\section{REFERENCES}

Bartroff, J. (2010). A Proof of the Bomber Problem's Spend-It-All Conjecture. To appear in Sequential Analysis.

Bartroff, J., Goldstein, L., Rinott, Y., and Samuel-Cahn, E. (2010). On Optimal Allocation of a Continuous Resource Using an Iterative Approach and Total Positivity. Advances in Applied Probability 42.

Klinger, A. and Brown, T. A. (1968). Allocating Unreliable Units to Random Demands. In Stochastic Optimization and Control, H. F. Karreman, ed., number 20 in Mathematics Research Center, U.S. Army, at the University of Wisconsin, Madison, pp. 173-209. New York: Wiley.

Samuel, E. (1970). On Some Problems in Operations Research. Journal of Applied Probability 7: 157-164.

Shepp, L. A., Simons, G., and Yao, Y.-C. (1991). On a Problem of Ammunition Rationing. Advances in Applied Probability 23: 624-641.

Simons, G. and Yao, Y.-C. (1990). Some Results on the Bomber Problem. Advances in Applied Probability 22: 412-432.

Weber, R. (1985). On a Problem of Ammunition Rationing. In Conference Report: Stochastic Dynamic Optimization and Applications in Scheduling and Related Areas, p. 148. Universität Passau, Fakultät für Mathematik und Informatik. Abstract. 